\title{
NONLINEAR FREDHOLM MAPS OF INDEX ZERO AND THEIR SINGULARITIES
}

\author{
R. A. PLASTOCK
}

\begin{abstract}
Let $F: X \rightarrow Y$ be a $C^{1}$ Fredholm map of index zero between two Banach spaces. Defining the singular set $B=\left\{x \mid F^{\prime}(x)\right.$ is not surjective $\}$, we study the local and global effect of $B$ on the map $F$. In particular it is shown that if $b \in B$ is isolated in $B$, then, for $\operatorname{dim} X$ and $\operatorname{dim} Y>3, F$ is a local homeomorphism at $b$. We then show that if $B$ consists of discrete points, $F$ is a global homeomorphism of $X$ onto $Y$. A nonlinear partial differential equation is included as an illustration.
\end{abstract}

When one formulates nonlinear elliptic partial differential equations as maps $F: X \rightarrow Y$ between Banach spaces we often encounter the class of $C^{1}$ Fredholm maps of index zero [4]. An immediate question is the existence and uniqueness of solutions, i.e., when is $F$ a global homeomorphism. If the singular set $B=\left\{x \mid F^{\prime}(x)\right.$ not surjective $\}$ is empty, then the inverse function theorem guarantees that $F$ is a local homeomorphism. In 1934 Banach and Mazur [1] showed that if in addition $F$ is proper (Definition 1), then $F$ is a global homeomorphism. We can ask for an extension of this result when $B$ is nonempty.

In §I we establish some preliminary results and definitions, including a proof of the Banach-Mazur theorem for completeness. In \$II we study the effects of the singular set and prove generalizations of both the inverse function theorem and the Banach-Mazur Theorem when $B \neq \varnothing$ (Theorems 4 and 5). We end with a nonlinear partial differential equation illustrating our results.

My thanks to M. S. Berger for his helpful suggestions.

I. Preliminaries. Let $F: X \rightarrow Y$ be a continuously differentiable map between two Banach spaces. We also suppose that $F$ is a Fredholm map [4].

The singular set $B$ of $F$ is defined as: $B=\left\{x \mid F^{\prime}(x)\right.$ is not surjective $\}$. Its image $S=F(B)$ is called the singular values. Since the noninvertible linear maps are closed in $L(X, Y)$ (the space of linear maps of $X$ into $Y$ ) then it follows from the continuity of the derivative map $F^{\prime}$ that $B$ is a closed set.

Since the conditions of the inverse function theorem require that $F^{\prime}(x)$ be invertible, we can reformulate this by saying that $F$ is a $C^{1}$ Fredholm map of

Received by the editors March 11, 1977 and, in revised form, April 11, 1977 and June 28, 1977. AMS (MOS) subject classifications (1970). Primary 47H15, 46N05; Secondary 35J60, 57045. Key words and phrases. Fredholm map of index zero, covering space map, proper. 
index $=0$ and $B=\varnothing$. Thus we shall also restrict ourselves to the class index $F=0$.

In order to globalize our results, we shall only consider what are called proper maps.

Definition 1. $F: X \rightarrow Y$ is proper if whenever $K \subset Y$ is compact then $F^{-1}(K)$ is compact in $X$.

In particular if $y$ is a point, then $F^{-1}(y)$ is certainly compact.

Also a proper map is a closed map for if $C \subset X$ is closed and the sequence $y_{n} \in F(C)$ converges to $y$, then $F^{-1}\left(\left\{y_{n}\right\} \cup y\right)$ is compact and so has a limit point $x$. By continuity $F(x)=y$.

Fundamental to our investigation is the notion of a covering space map.

Definition 2. A map $F$ of $X$ onto $Y$ is a covering space map if we can find a covering of $Y$ by open sets $U$ so that for each $U, F^{-1}(U)$ is the disjoint union of open sets each of which is mapped homeomorphically onto $U$.

The key to our use of covering space maps is [5]:

LEMMA 1. If $F: X \rightarrow Y$ is a covering space map, $X$ and $Y$ pathwise connected and $Y$ simply connected, then $F$ is a global homeomorphism.

Thus our first question is to find conditions which insure that a map is a covering space map. This is answered by [3]:

Covering Space Theorem 1. Let $U \subset X$ be a connected open set in a Banach space $X$. Then $F: U \rightarrow F(U)$ is a covering space map between these two sets if (i) $F$ is a local homeomorphism and (ii) $F$ is proper.

Before we discuss the effects that the singular set has on the properties of a mapping we shall need to know what happens when there is no singular set, i.e., when $B=\varnothing$. Locally the inverse function theorem tells us that the map is a local homeomorphism. The question is what happens globally. In this case the definitive result is the Banach-Mazur theorem [1]:

THEOREM 2. In order that $F: X \rightarrow Y$ be a global homeomorphism of $X$ onto $Y$ it is necessary and sufficient that (i) $F$ is a proper map and (ii) $F$ is a local homeomorphism.

Proof. The necessity follows from the fact that a homeomorphism is proper since $F^{-1}$ is continuous. The sufficiency follows, once we show that $F$ maps $X$ onto $Y$, from Theorem 1 with $U=X$ and Lemma 1 observing that a Banach space $Y$ is certainly connected and simply connected. However the ontoness of $F$ is assured since (i) a proper map is in particular a closed map and (ii) a local homeomorphism is an open map. Thus $F[X]$ is both open and closed and so must be all of $Y$ by connectivity.

When $F$ is continuously differentiable we can reformulate this theorem using the inverse function theorem.

THEOREM 2a. In order that the $C^{1}$ map $F: X \rightarrow Y$ be a global diffeomorphism, it is necessary and sufficient that (i) $F$ is proper, (ii) $F$ is a 
Fredholm map of index $=0$ and (iii) $B=\varnothing$.

II. The singular set. The Banach-Mazur theorem (our Theorems 2 and 2a) leads us to ask how the singular set affects the conclusions of this theorem, in particular what type of singular set insures that our map is a global homeomorphism.

THEOREM 3. Let $F: X \rightarrow Y$ be a $C^{1}$ proper Fredholm map of index $=0$. Then if (i) $F(X) \neq S$ and (ii) $Y-S$ is connected then $F: X-F^{-1}(S) \rightarrow Y-S$ is a covering space map.

Proof. Since $F$ is proper and $B$ is closed, $S=F(B)$ is closed. Thus $X-F^{-1}(S)$ and $Y-S$ are open. Since $F^{\prime}(x)$ is invertible on $X-F^{-1}(S)$, we can apply the inverse function theorem to conclude that $F$ is an open map on this set.

We next show that $F$ is a proper map between $X-F^{-1}(S)$ and $Y-S$. This is enough because $F$ will then be an open and closed map and so by the connectivity of $Y-S$, it will map $X-F^{-1}(S)$ onto $Y-S$. We may then apply Theorem 1 to obtain the desired conclusion.

However $F$ is proper on $X-F^{-1}(S)$, for if $K \subset Y-S$ is compact then $K$ is compact in $Y$. Thus $F^{-1}(K)$ is compact in $X$. However $F^{-1}(K) \subset X-$ $F^{-1}(S)$ and so it is compact in this set.

We observe that with $F$ and $S$ satisfying the conditions in the above theorem, then $F$ maps $X$ onto $Y$ since the theorem shows it maps $X-$ $F^{-1}(S)$ onto $Y-S$, and trivially, $F^{-1}(S)$ onto $S$. Thus if $S$ is "thin" enough, the surjectivity of $F$ is insured and the study of $F$ reduces to its nice behavior as a covering map on $X-F^{-1}(S)$ onto $Y-S$ and its bad behavior on $F^{-1}(S)$ onto $S$. In the latter case however, $F$ is still a proper map. With further assumptions, we can study $F$ on this bad part. In fact we show that isolated singularities are removable in the following sense:

TheOREM 4. Let $F: X \rightarrow Y$ be a $C^{\prime}$ proper Fredholm map of index $=0$ between two Banach spaces whose dimensions are 3 or greater. If $b \in B$ is isolated in $B$ then $F$ is a local homeomorphism about $b$.

Proof. Choose an open ball $O$ about $b$ so that $\bar{O} \cap B=b$. Let $F(b)=p$. If $x \in F^{-1}(p) \cap \bar{O}$ and $x \neq b$ then $F$ is a local homeomorphism about $x$. Thus $b$ is the only limit point of $F^{-1}(p) \cap \bar{O}$. Hence $F^{-1}(p) \cap \partial O$ is compact and so consists of only a finite number of points. Thus by enclosing these points in sufficiently small closed balls and then excising these closed balls from $O$, we construct an open set $O^{\prime}$ about $b$ such that $\partial O^{\prime} \cap F^{-1}(p)$ $=\varnothing$.

About $p$ choose open balls $O_{n}$ of radius $1 / n$. Let $C_{n}$ be the component of $F^{-1}\left(O_{n}\right) \cap O^{\prime}$ containing $b$. For some $N, \bar{C}_{N} \cap \partial O^{\prime}=\varnothing$. If not then we can find a sequence $x_{n} \in C_{n} \cap \partial O^{\prime}$. Since $F\left(x_{n}\right) \in \bar{O}_{n}$, then $F\left(x_{n}\right) \rightarrow p$. By the properness of $F, x_{n} \rightarrow \bar{x}$ and $\bar{x} \in F^{-1}(p) \cap \partial O^{\prime}$. This contradicts our construction of $O^{\prime}$. Thus $\bar{C}_{N} \subset O^{\prime}$ for some $N$. 
We show that $F: C_{N} \rightarrow O_{N}$ is proper. Let $K \subset O_{N}$ be compact. Thus $K$ is compact in $Y$ and so $F^{-1}(K) \cap \bar{C}_{N}$ is compact and furthermore it does not intersect $\partial O^{\prime}$. Hence $\operatorname{dist}\left(F^{-1}(K) \cap \bar{C}_{N}, \partial O^{\prime}\right)=\varepsilon>0$ and so if $x \in F^{-1}(K)$ $\cap \bar{C}_{N}$ there is an open ball $O_{x}$ about it so that $\bar{O}_{x} \subset O^{\prime}$ and $F\left(O_{x}\right) \subset O_{N}$ (otherwise we could find a sequence $x_{n} \in F^{-1}(K) \cap \bar{C}_{N}, x_{n} \rightarrow x$ and $F\left(x_{n}\right)$ $\notin O_{N}$. However since $F\left(x_{n}\right) \rightarrow F(x)$, this would contradict the fact that $F(x)$ is an interior point of $\left.O_{N}\right)$. Thus $O_{x} \subset O^{\prime} \cap F^{-1}\left(O_{N}\right)$. Since $O_{x}$ is connected and $O_{x} \cap C_{N} \neq \varnothing$, then $O_{x} \subset C_{N}$. Hence $\bar{C}_{N} \cap F^{-1}(K)=C_{N} \cap F^{-1}(K)$ is compact. Thus $F$ is proper on $C_{N}$ into $O_{N}$.

Next we show that $F: C_{N}-F^{-1}(p) \rightarrow O_{N}-p$ is a homeomorphism. This follows from Theorem 3 and Lemma 1 after we check their hypotheses. Firstly, $F$ is a proper map by the above argument. It is also open since there are no singular points. Hence $F$ is onto by the connectivity of $O_{N}-p$. Secondly $C_{N}-F^{-1}(p)$ is connected. This follows in the case of infinite dimensional $X$ [8] since $F^{-1}(p) \cap C_{N}$ is compact and in the finite dimensional and thus separable case since $F^{-1}(p) \cap C_{N}$ is countable and compact. Finally if $\operatorname{dim} Y \geqslant 3, O_{N}-p$ is simply connected.

We finally show that $F$ is $1-1$ on $C_{N}$ and thus a homeomorphism by the invariance of domain theorem [6]. We shall show that $F^{-1}(p) \cap C_{N}=b$.

Suppose $r$ is a regular value of $F^{-1}(p) \cap C_{N}$. Then $r$ and $b$ are contained in disjoint open sets $O_{r}$ and $O_{b}$ in $C_{N}$. Furthermore we choose $O_{r}$ so that $F$ is a homeomorphism on it. Choosing a sequence $x_{n} \in C_{N}-F^{-1}(p)$ and $x_{n} \rightarrow b$, we can find a sequence $\bar{x}_{n} \rightarrow r$ and $x_{n} \neq \bar{x}_{n}, F\left(x_{n}\right)=F\left(\bar{x}_{n}\right)$ contradicting the univalence of $F$ on $C_{N}-F^{-1}(p)$. Hence $F^{-1}(p) \cap C_{N}=b$.

The counterexamples $F(x)=x^{2}$ in $\mathbf{R}^{\prime}$ and $F(z)=z^{2}$ in $\mathbf{R}^{2}$ illustrate the necessity of the restrictions on the dimensions of the spaces in Theorem 4.

In [6] it is shown that if $F$ is a $1-1$ proper Fredholm map with index $=0$, then $B$ is nowhere dense. We present a partial converse by applying Theorems 4 and 2.

TheOREM 5. Let $F: X \rightarrow Y$ be a $C^{\prime}$ proper Fredholm map of index 0 between Banach spaces whose dimensions are $\geqslant 3$. If the singular set $B$ is discrete, then $F$ is a global homeomorphism of $X$ onto $Y$.

Theorem 4 essentially shows that at an isolated singularity $b$, locally $F^{-1}(F(b))=b$. We globalize this relation in the case where $X$ and $Y$ are infinite dimensional.

THEOREM 6. Let $F: X \rightarrow Y$ be a $C^{\prime}$ proper Fredholm map of index 0 between infinite dimensional Banach spaces. If the singular set $B$ is the countable union of compact sets, then $F^{-1}(F(B))=B$, and $F$ is a global diffeomorphism of $X-B$ onto $Y-S$.

Proof. Since $F$ is proper, $S=F(B)$ and $F^{-1}(S)$ are closed and the countable union of compact sets. By the infinite dimensionality of $X$ and $Y$, $X-F^{-1}(S)$ is connected and $Y-S$ is connected and simply connected [8]. By Theorem 3 and Lemma $1 F$ is a global diffeomorphism of $X-F^{-1}(S)$ 
onto $Y-S$. Let $y \in S$. We look at $F^{-1}(y)$. Let $b \in F^{-1}(y) \cap B$. Suppose $r \in F^{-1}(y)$ is a regular point. Then $r$ and $b$ are isolated from each other and can be enclosed in disjoint open sets $O_{r}$ and $O_{b}$. We choose $O_{r}$ so that $F$ is a homeomorphism on it. Since $F^{-1}(S)$ is of the first category in $X$, we can find a sequence $x_{n} \rightarrow b$ and $x_{n} \in X-F^{-1}(S) \cap O_{b}$. Since $F\left(x_{n}\right) \rightarrow y$, we can find points $\bar{x}_{n} \rightarrow r$ and $F\left(x_{n}\right)=F\left(\bar{x}_{n}\right)$, contradicting the univalence of $F$ on $X-F^{-1}(S)$. Hence $F^{-1}(S)$ contains no regular points, i.e., $F^{-1}(S)=B$.

We remark again that Theorem 6 is purely infinite dimensional as proven. Thus the size of the space proves to be an advantage to us. I do not know if Theorem 6 is true in finite dimensions.

As an illustration of our results, let us look at the equation:

$$
\begin{aligned}
& \Delta u+\lambda_{1} u-u^{3}=f\left(f \in L^{2}\right) . \\
& u=0 \text { on the boundary of } \Omega .
\end{aligned}
$$

Here $\Omega$ is a bounded domain in $\mathbf{R}^{N}$ and $\lambda_{1}$ is the smallest positive eigenvalue of $\Delta$ on $\Omega$. We reformulate $(*)$ as a map $F(u)$ on the Sobolev space $\dot{W}_{1,2}$ where $(F(u), v)_{1,2}=\int \nabla u \cdot \nabla v-\lambda_{1} \int u v+\int u^{3} v$. In order to avoid excessive detail we assume $N \leqslant 3$ is chosen so that results involving the Sobolev imbedding theorems and regularity of solutions are justified. We refer the reader to [2] for a treatment of techniques and theorems used in the functional analytic approach to partial differential equations.

On $\dot{W}_{1,2} F(u)$ has a derivative $F^{\prime}(u) v=\Delta v+\lambda_{1} v-u^{2} v$. Thus $F^{\prime}(u)$ is a selfadjoint elliptic operator and so $F(u)$ is a Fredholm map of index zero. Also if $F^{\prime}(u) v=0$, then using the variational characterization of $\lambda_{1}$, we have $0=\left(F^{\prime}(u) v, v\right)=\int|\nabla v|^{2}-\lambda_{1} v^{2}+\int u^{2} v^{2}>0$ unless $u=0$. In this case $F^{\prime}(0) v=0$ is the problem $\Delta v+\lambda_{1} v=0, v=0$ on the boundary of $\Omega$. Since this problem has nontrivial solutions we conclude that for $F(u)$, the singular set $B=\{0\}$.

We now show $F$ is proper. First the inverse image of a bounded set is bounded. To see this we prove the equivalent statement: $\|F(u)\|_{1,2} \rightarrow \infty$ as $\|u\|_{1,2} \rightarrow \infty$. So suppose $\|u\|_{1,2}^{2}=\|\nabla u\|_{2}^{2}+\|u\|_{2}^{2} \rightarrow \infty$. If $\|\nabla u\|_{2}^{2} \rightarrow \infty$ and $\|u\|_{2}^{2} \leqslant k$, then using the fact that $\|\nabla u\|_{2}^{2} \geqslant c\|u\|_{2}^{2}$ we hae $(F(u), u)=\int|\nabla u|^{2}$ $-\lambda_{1} u^{2}+\int u^{4} \geqslant\|\nabla u\|_{2}^{2}-\lambda_{1} k$. Thus for $\|u\|_{1,2}^{2}$ large enough, $(F(u), u) /\|u\|_{1,2}^{2}$ $\geqslant \gamma>0$. If $\|u\|_{2}^{2} \rightarrow \infty$ also, then $(F(u), u) \geqslant\|\nabla u\|_{2}^{2}+\left(\beta\|u\|_{2}^{2}-\lambda_{1}\right)\|u\|_{2}^{2}$ (since $L^{4} \subset L^{2}$ ). Thus if $\|u\|_{1,2} \rightarrow \infty$, then $(F(u), u) /\|u\|_{1,2}^{2} \geqslant \varepsilon>0$.

Applying the Cauchy-Schwarz inequality we can conclude that $\|F(u)\|_{1,2} \rightarrow$ $\infty$ when $\|u\|_{1,2} \rightarrow \infty$.

So to show $F$ is proper, let $K \subset \dot{W}_{1,2}$ be compact. By the above result $F^{-1}(K)$ is bounded. Thus if $u_{n}$ is a sequence in $F^{-1}(K)$, then $\left\{u_{n}\right\}$ has a weakly convergent subsequence since $\dot{W}_{1,2}$ is a Hilbert space. As $F\left(u_{n}\right)$ lies in the compact set $K$, it has a convergent subsequence. Renumbering, we have $u_{n} \rightarrow u$ weakly and $F\left(u_{n}\right) \rightarrow y$ strongly. However if $N \leqslant 3$, we can apply the Sobolev theorems governing compact imbeddings to conclude that $u_{n} \rightarrow u$ strongly in $L^{2}$ and $L^{4}$. Thus since $\left(F\left(u_{n}\right), u_{n}\right)$ converges and since $\left(F\left(u_{n}\right)\right.$, 
$\left.u_{n}\right)=\left\|\nabla u_{n}\right\|_{2}^{2}-\lambda_{1}\left\|u_{n}\right\|_{2}^{2}+\left\|u_{n}\right\|_{4}^{4}$, then we conclude that $\left\|\nabla u_{n}\right\|_{2}$ converges. Thus we are in the situation where $u_{n}$ converges weakly and $\left\|u_{n}\right\|_{1,2}$ converges in a Hilbert space. Thus $u_{n} \rightarrow u$ strongly, i.e., every sequence in $F^{-1}(K)$ has a convergent subsequence. Thus $F$ is proper.

We now apply Theorem 5 to conclude that $F$ is a global homeomorphism of $\stackrel{\circ}{W}_{1,2}$ onto itself, and so our problem (*) has, for every $f \in L^{2}$, a unique solution depending continuously on $f$.

A natural question to ask is for generalizations to Fredholm maps with index $p>0$. However in this case the results are radically different. For example it can be shown that there is no equivalent statement of the Banach-Mazur Theorem 2a. In fact if $F$ is a proper $C^{1}$ Fredholm map with positive index, then $B$ cannot be empty, i.e., such maps always have singularities. This result and a general investigation of the positive index case will appear in [7].

\section{REFERENCES}

1. S. Banach and S. Mazur, Über mehrdeutige stetige Abbildungen, Studia Math. 5 (1934), 174-178.

2. M. S. Berger, Nonlinearity and functional analysis, Academic Press, New York, 1977.

3. R. Plastock, Homeomorphisms between Banach spaces, Trans. Amer. Math. Soc. 200 (1974), 169-183.

4. S. Smale, An infinite dimensional version of Sard's theorem, Amer. J. Math. 87 (1965), 861-866.

5. E. H. Spanier, Algebraic topology, McGraw-Hill, New York, 1966.

6. A. J. Tromba, Some theorems on Fredholm maps, Proc. Amer. Math. Soc. 34 (1972), 578-585.

7. M. S. Berger and R. Plastock, On the singularities of nonlinear Fredholm operators, Bull. Amer. Math. Soc. 83 (1977), 1316-1318.

8. W. H. Cutler, Negligible subsets of infinite dimensional Fréchet manifolds, Proc. Amer. Math. Soc. 23 (1969), 668-675.

Department of Mathematics, New Jersey Institute of Technology, Newark, New JERSEY 07102 